\title{
$\mathrm{PbTiO}_{3} / \mathrm{SrTiO}_{3}(010)$ 界面位错与电子富集的研究
}

\author{
吴凯 \\ 北京大学化学与分子工程学院, 北京 100871
}

\section{Characterization of Dislocation and Electron Aggregation at (010) $\mathrm{PbTiO}_{3} / \mathrm{SrTiO}_{3}$ Heterointerface}

\author{
Kai Wu \\ College of Chemistry and Molecular Engineering, Peking University, Beijing 100871, P. R. China. \\ Email: kaiwu@pku.edu.cn. \\ Published online: June 2, 2020.
}

\section{1 背景介绍}

钙铁矿氧化物界面的物理特性, 可以通过晶 格匹配、应力、极化场等参数进行有效的调控, 组 合多种优异性质以实现复合功能与多场调控。在 铁性异质结材料中, 晶格缺陷也可以作为界面调 控的手段之一。例如, 位错附近的晶格畸变会使铁 电材料产生局域应变场, 造成局域自发极化强度 的显著变化 ${ }^{1}$ 。氧化物材料中, 缺陷核心区域不仅 展示出晶体结构和化学组分的改变(如氧浓度), 局 域晶格的畸变也会导致不同于基体本身的新性能 出现, 比如导电性等 ${ }^{2}$ 。在铁电材料中, 缺陷的类 型、分布、密度等特性不仅影响铁电畴界面的形成 和结构, 而且影响异质薄膜中界面序参量的耦合 机制。因此, 研究铁电氧化物异质界面以及界面位 错区域的原子结构与电子结构, 对于优化、改进材 料体系以及加深对材料物理机理的理解十分必要。

当前, 有关于铁电材料异质界面上位错的存 在, 对铁电畴结构等相关物理性能的影响机制, 尚 未有公认的理论与实验认知体系。例如, Jia等人在 2009年发现纳米尺寸的 $\mathrm{PbZr}_{0.2} \mathrm{Ti}_{0.8} \mathrm{O}_{3}$ 薄膜界面上的 位错, 会诱导发生局域性的自发极化减小现象 3 。而 与之相反的是, $\mathrm{Wu}$ 等人在 2013 年通过模拟计算发 现单晶 $\mathrm{PbTiO}_{3}$ 内的位错会增强铁电极化 ${ }^{4}$ 。而球差 校正透射电镜以及能量单色器等技术的发展, 为 原子尺度下研究材料原子结构信息和电子结构信 息提供了一个强有力的工具 ${ }^{5-9}$ 。浙江大学材料科 学与工程学院田鹤研究员课题组利用原子分辨球 差校正透射电镜与电子能量损失谱, 对
$\mathrm{PbTiO}_{3} / \mathrm{SrTiO}_{3}(010)$ 异质界面进行了表征, 分析了 该界面上周期性存在的失配位错的原子结构, 以 及位错核及其附近区域的电荷分布, 探究了该电 荷富集的来源。该工作已在物理化学学报上在线 发表(doi: 10.3866/PKU.WHXB201906019) ${ }^{10}$ 。

\section{2 研究亮点}

(1)利用原子级HAADF-STEM成像, 对单畴 $\mathrm{PbTiO}_{3}$ 为基底静电力驱动生长的 $(001)$ 晶 $\mathrm{PbTiO}_{3} / \mathrm{SrTiO}_{3}$ 界面的原子结构进行了表征分析。 发现了在该界面周期性存在柏格斯矢量为 $a$ [001] 的失配位错, 刃形位错多余原子面均位于 $\mathrm{SrTiO}_{3}$ 一 侧, 周期约为 $8.5 \mathrm{~nm}$ 。

(2)采用原子级分辨率与高能量分辨率的电子 能量损失谱对位错核心区域的电荷分布进行了探 究。结果表明该区域存在电子富集与氧空位耗尽 现象, 而富集电子来源于 $\mathrm{PbTiO}_{3}$ 内部的氧空位。

\section{3 图文解析}

\section{要点1 周期性失配位错对于异质界面失配应力 的释放}

具有较大失配的两种材料在形成异质界面 时, 由于较大的失配应力的存在, 难以形成高质量 原子级平整的外延薄膜。如图1所示, $\mathrm{PbTiO}_{3} 、 \mathrm{SrTiO}_{3}$ 两种材料 $(010)$ 晶面约为 $6.4 \%$ 的失配度, 使得目前 在文献中尚未有报道 $\mathrm{PbTiO}_{3} / \mathrm{SrTiO}_{3}(010)$ 薄膜体系 的外延生长。周期性出现的失配位错, 其柏格斯矢 量为 $a[001]$, 在 $\mathrm{STO}$ 一侧周期性出现了多余原子 

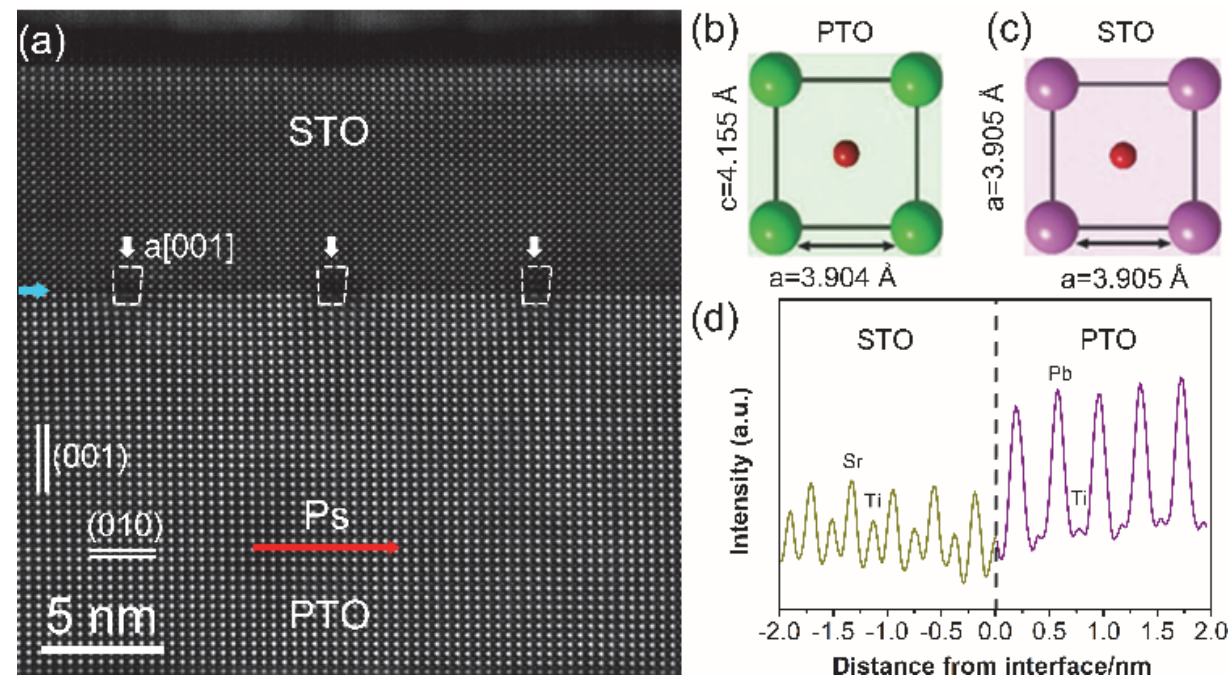

图1 (010)晶面 $\mathrm{PbTiO}_{3} / \mathrm{SrTiO}_{3}$ 异质界面的截面HAADF-STEM表征。

面, 起到了失配缓解的作用, 满足了形成原子级平 整异质结的先决条件。

要点 2 失配位错核心区域EELS Ti $L$ 吸收边表征 材料内部或界面上存在的缺陷态, 比如失配 位错, 往往会对局域电子结构产生影响。电子能量 损失谱(EELS)的 Ti $L$ 吸收边可以用来分析局域出 现的电子结构变化。如图2所示, 位错核心下方局 部区域, Ti $L$ 吸收边 $t_{2 \mathrm{~g}}-e_{\mathrm{g}}$ 辟裂值呈现明显的减小特 征，位错核心对应的蓝色区域䢃裂值 $1.48 \mathrm{eV}$, 明 显小于PTO正常值 $1.60 \mathrm{eV}$, 该特征意味着体系内 有 $\mathrm{Ti}^{4+}$ 到 $\mathrm{Ti}^{3+}$ 的价态改变, 可观的 3 价 $\mathrm{Ti}$ 组分, 或有
负电荷即电子富集。

要点3 位错核心区域的原子尺度价态分析

作者进一步对单一位错核区域进行了原子级 分辨率以及高能量分辨率的电子能量损失谱分 析。采用标准谱线性拟合的方法, 得到了原子级 的价态分布图。如图3所示, 在位错核处 10个单胞 的区域, $\mathrm{Ti}^{4+}$ 具有更低的组分, $\mathrm{Ti}^{3+}$ 具有更高的组 分。通过价态换算富集电子量, 可计算出在位错核 心处的电子浓度约为 $1.6 \times 10^{14} \mathrm{~cm}^{-2}$ 。对 $\mathrm{O} K$ 精细结 构的分析表明, 电子来源于 $\mathrm{PbTiO}_{3}$ 材料内部的氧 空位。
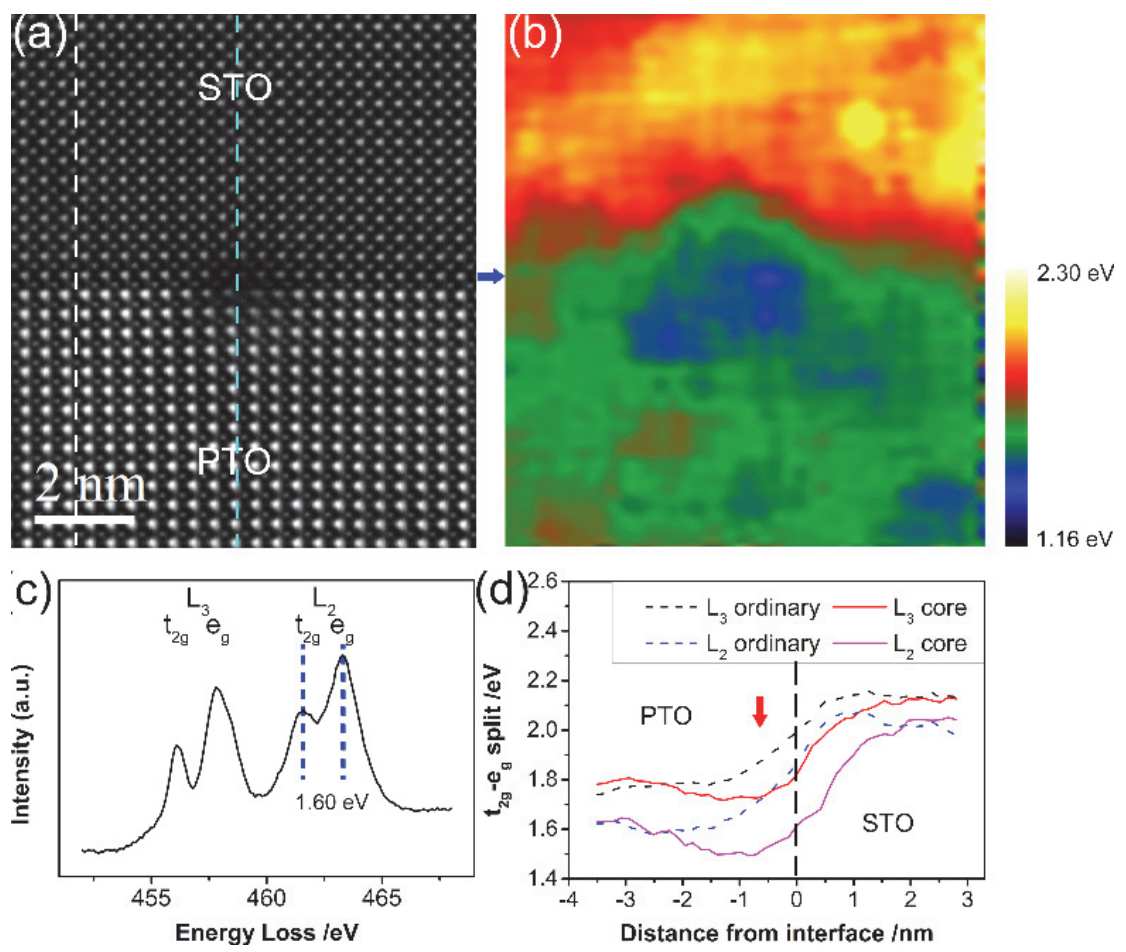

图2 位错核附近区域EELS Ti $L$ 吸收边表征。 

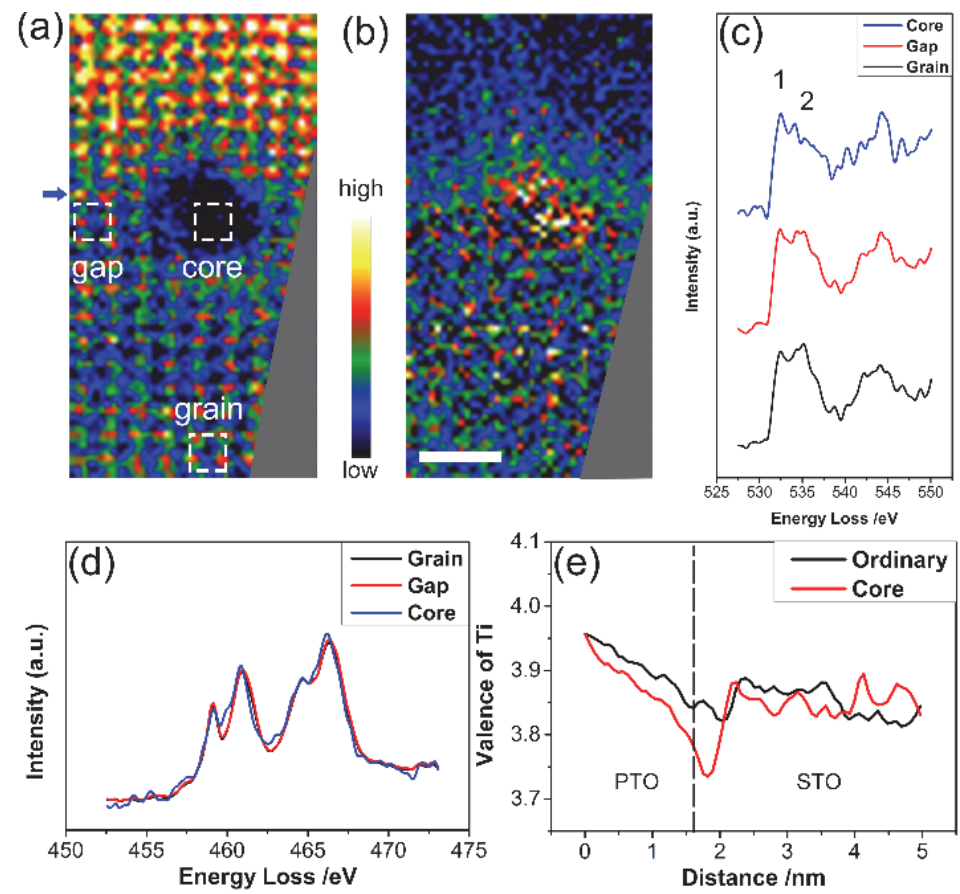

图3 位错核附近区域原子尺度价态表征。

\section{4 全文小结}

该工作报道了 $(001)$ 晶面 $\mathrm{PbTiO}_{3} / \mathrm{SrTiO}_{3}$ 界面上 周期出现的失配位错, 其位错核心区域存在富集 的电子，同时发现了该电子的可能来源为 $\mathrm{PbTiO}_{3}$ 内部的氧空位。作者推测该一维分布的电子, 有可 能对绝缘材料在位错线上电子电导率的提升起到 关键作用。该工作对于探索界面位错对铁电氧化 物界面的电学性能调控, 以及氧化物界面处电荷 的一维分布等, 具有启发意义。

\section{References}

(1) Chu, M. -W.; Szafraniak, I.; Scholz, R.; Harnagea, C.; Hesse, D.; Alexe, M.; Gösele, U. Nat. Mater. 2004, 3, 87. doi: $10.1038 /$ nmat 1057

(2) Szot, K.; Bihlmayer, G.; Speier, W. Nature of the Resistive Switching Phenomena in $\mathrm{TiO}_{2}$ and $\mathrm{SrTiO}_{3}$ : Origin of the Reversible InsulatorMetal Transition. In Solid State Physics; Camley, R. E., Stamps, R. L., Eds.; Academic Press: Cambridge, MA, USA, 2014; Vol. 65, Chapter Four, pp. 353-559.

(3) Jia, C. L.; Mi, S. B.; Urban, K.; Vrejoiu, I.; Alexe, M.; Hesse, D. Phys. Rev. Lett. 2009, 102, 117601. doi: 10.1103/PhysRevLett.102.117601

(4) Wu, H. H.; Wang, J.; Cao, S. G.; Zhang, T. Y. Appl. Phys. Lett. 2013, 102, 232904. doi: 10.1063/1.4809945

(5) Sawada, H.; Hosokawai, F.; Kaneyama, T.; Ishizawa, T.; Terao, M.; Kawazoe, M.; Sannomiya, T.; Tomita, T.; Kondo, Y.; Tanaka, T.; et al. Jpn. J. Appl. Phys. Part 2-Lett. Expr. Lett. 2007, 46, L568. doi: $10.1143 /$ jjap.46.1568

(6) Muller, D. A.; Kourkoutis, L. F.; Murfitt, M.; Song, J. H.; Hwang, H. Y.; Silcox, J.; Dellby, N.; Krivanek, O. L. Science 2008, 319, 1073. doi: $10.1126 /$ science. 1148820

(7) Essers, E.; Benner, G.; Mandler, T.; Meyer, S.; Mittmann, D.; Schnell, M.; Hoeschen, R. Ultramicroscopy 2010, 110, 971 doi: 10.1016/j.ultramic.2010.02.009

(8) Krivanek, O. L.; Ursin, J. P.; Bacon, N. J.; Corbin, G. J.; Dellby, N.; Hrncirik, P.; Murfitt, M. F.; Own, C. S.; Szilagyi, Z. S. Philosoph. Trans. Royal Soc. 2009, 367, 3683. doi: 10.1098/rsta.2009.0087

(9) Rose, H. H. J. Electron Micros. 2009, 58, 77. doi: 10.1093/jmicro/dfp012

(10) Chen, X.; Tian, H.; Zhang, Z. Acta Phys. -Chim. Sin. 2020, 36, 1906019. [陈星, 田鹤, 张泽. 物理化学学报, 2020, 36, 1906019.] doi: 10.3866/PKU.WHXB201906019 